\title{
Multimode and Long-Lived Quantum Correlations Between Photons and Spins in a Crystal
}

\author{
Cyril Laplane, Pierre Jobez, Jean Etesse, Nicolas Gisin, and Mikael Afzelius ${ }^{\dagger}$ \\ Groupe de Physique Appliquée, Université de Genève, CH-1211 Genève 4, Switzerland
}

(Received 2 February 2017; published 24 May 2017)

\begin{abstract}
The realization of quantum networks and quantum repeaters remains an outstanding challenge in quantum communication. These rely on the entanglement of remote matter systems, which in turn requires the creation of quantum correlations between a single photon and a matter system. A practical way to establish such correlations is via spontaneous Raman scattering in atomic ensembles, known as the DuanLukin-Cirac-Zoller (DLCZ) scheme. However, time multiplexing is inherently difficult using this method, which leads to low communication rates even in theory. Moreover, it is desirable to find solid-state ensembles where such matter-photon correlations could be generated. Here we demonstrate quantum correlations between a single photon and a spin excitation in up to 12 temporal modes, in a ${ }^{151} \mathrm{Eu}^{3+}$-doped $\mathrm{Y}_{2} \mathrm{SiO}_{5}$ crystal, using a novel DLCZ approach that is inherently multimode. After a storage time of $1 \mathrm{~ms}$, the spin excitation is converted into a second photon. The quantum correlation of the generated photon pair is verified by violating a Cauchy-Schwarz inequality. Our results show that solid-state rare-earth-ion-doped crystals could be used to generate remote multimode entanglement, an important resource for future quantum networks.
\end{abstract}

DOI: 10.1103/PhysRevLett.118.210501

Remote entanglement between light and matter is commonly based on the creation of quantum correlations between the internal state of a spin system and an optical mode [1-3]. This is due to the fact that spins can function as a long-duration quantum memory for photons, which are ideal long-distance carriers of quantum information. Most schemes also rely on discrete quantum variables, i.e., a single spin excitation quantum correlated with a single photon propagating in the optical mode [3]. The DuanLukin-Cirac-Zoller (DLCZ) approach is one of the moststudied schemes for establishing such correlations, as it is based on relatively simple resources: an ensemble of atoms interacting with a coherent laser field [1]. The ensemble of atoms is polarized into its lowest spin state $|g\rangle$, cf. Fig. 1(a), then an off-resonant laser write pulse causes some spins to change their internal state to $|s\rangle$ through an optical Raman interaction. The creation of a spin excitation is strongly correlated to the emission of a corresponding Stokes photon in the optical mode.

The conventional DLCZ scheme has been widely implemented in atomic alkali-metal gases at the single-photon level [6-8], as well as for the entanglement of remote atomic ensembles [9] and for the realization of entanglement swapping between two pairs of nodes [10]. The scheme is, however, inherently single mode in time; hence, only a single spin-photon correlation can be generated and stored. Spatial multimode generation can be achieved [11], but the time domain remains very attractive as it is compatible with propagation through low-loss single-mode fibers.

Solid-state devices are gaining interest as potential quantum nodes for quantum networks. A large variety of solid-state systems are currently being investigated, among those single nitrogen-vacancy centers [12], quantum dots [13], mechanical oscillators [14], and ensembles of rare-earthmetal [15-19]. Rare-earth-ion-doped crystals are unique solid-state ensembles as they possess both long opticaland spin-coherence times, as well as large inhomogeneous linewidths in the optical domain [20]. The combination of long optical-coherence times (i.e., narrow homogeneous linewidths) and large inhomogeneous broadening implies that there is a large temporal multimode capacity [3].

Quantum memory schemes based on inhomogeneous transitions, such as the gradient echo memory [21] and atomic frequency comb (AFC) memory [22] schemes, have been successfully implemented in order to store multiple temporal modes in a single spatial mode, in alkali-metal gases [21] and rare-earth-ion-doped crystals [4], respectively. Yet, for DLCZ-type schemes, the use of inhomogeneous transitions for the generation of temporally multimode spin-photon correlations remains largely unexplored. Two recent experiments have demonstrated quantum correlations between light and spins in up to two temporal modes, in a laser-cooled ${ }^{87} \mathrm{Rb}$ gas [23] and a $\mathrm{Pr}^{3+}$-doped crystal [19], respectively, with storage times limited to the range 10-20 $\mu \mathrm{s}$. We also emphasize that in the latter experiment continuous variables states of light were used, which are extremely sensitive to propagation losses and require additional distillation techniques [24]. Conventional DLCZ schemes use single-photon states [1], however, as the loss of a photon does not increase the noise.

Here we present experimental results on the generation of nonclassical correlations between a single photon at $580 \mathrm{~nm}$ 
(a)
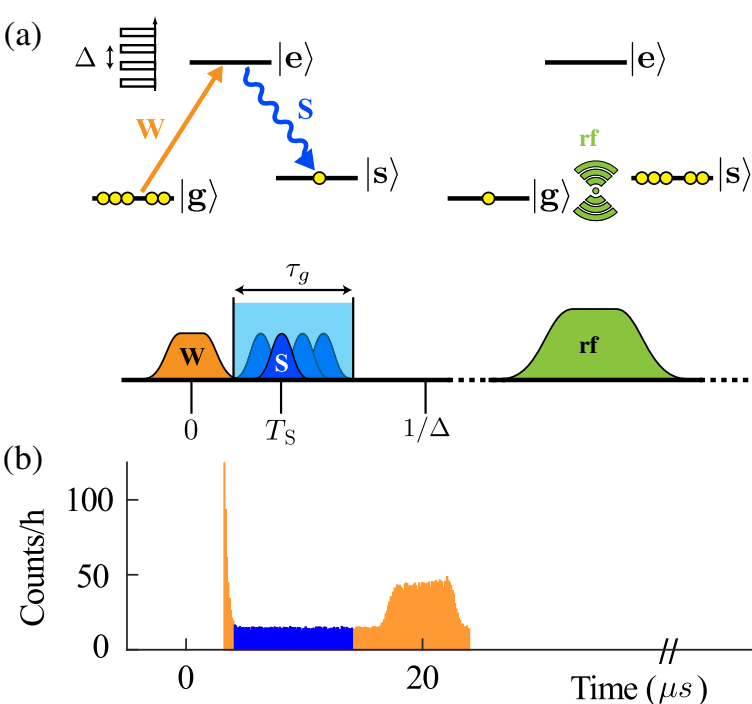

(b)
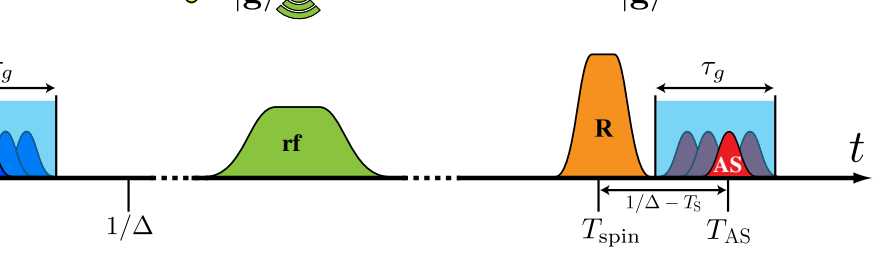

\begin{abstract}
$\Delta$
\end{abstract}

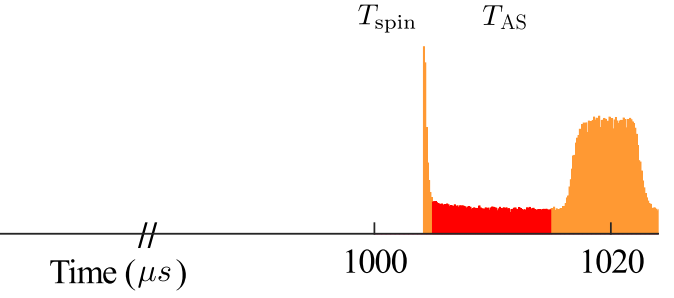

(c)

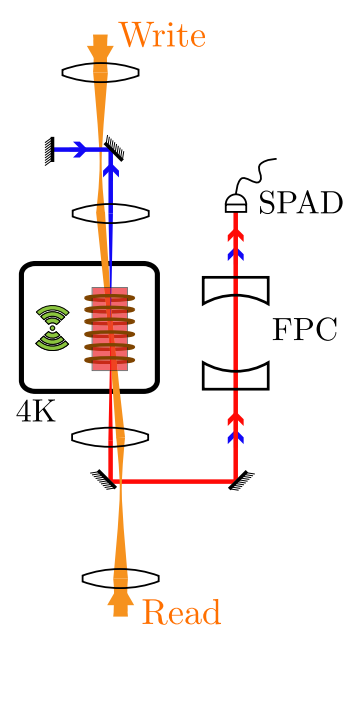

FIG. 1. The AFC-based DLCZ scheme and experimental setup. (a) We consider two spin ground states $|g\rangle,|s\rangle$ and an excited state $|e\rangle$ of the ${ }^{151} \mathrm{Eu}^{3+}$ ion in $\mathrm{Y}_{2} \mathrm{SiO}_{5}$. Initially $|s\rangle$ is emptied and a comb structure with periodicity $\Delta$ is prepared on the $|g\rangle \leftrightarrow|e\rangle$ transition using well-established optical pumping techniques [4]. A write pulse $(W)$ resonantly excites a small fraction $(<1 \%)$ of the ions to $|e\rangle$ and the detection of a single spontaneous Stokes emission (S) heralds a single excitation in $|s\rangle$. A sequence of radiofrequency (rf) pulses resonant with the $34.5 \mathrm{MHz}|g\rangle \leftrightarrow|s\rangle$ spin transition extends the storage time to $T_{\text {spin }}=1000 \mu \mathrm{s}$ [5]. The sequence contains an uneven number of pulses, which effectively inverts the population. A read pulse $(R)$ excites the stored spin excitation to $|e\rangle$, which leads to a strong collective emission of an anti-Stokes photon (AS) at a time $T_{\mathrm{AS}}$ such that $T_{\mathrm{S}}+T_{\mathrm{AS}}=T_{\mathrm{spin}}+1 / \Delta$. Ideally the read pulse should be a population-inversion pulse covering the entire spectral bandwidth induced by the weaker write pulse. (b) An example of a photon-counting histogram showing the Stokes (blue region) and antiStokes (red region) detection gates. The pulses at $20 \mu$ s and $1020 \mu$ s are AFC echoes produced by the write and read pulses. (c) The crystal is put in a low-vibration cryostat at $4 \mathrm{~K}$. A coil is used to apply the rf sequence (in green). The write and read beams are counterpropagating $\left(\mathbf{k}_{W}=-\mathbf{k}_{R}\right.$ ), and have a small angle (about $3^{\circ}$ ) with respect to the Stokes and anti-Stokes modes $\left(\mathbf{k}_{\mathrm{S}}\right.$ and $\mathbf{k}_{\mathrm{AS}}$ ). The phase-matching condition $\mathbf{k}_{W}+\mathbf{k}_{R}-\mathbf{k}_{\mathrm{S}}-\mathbf{k}_{\mathrm{AS}}=0$ then implies that $\mathbf{k}_{\mathrm{S}}=-\mathbf{k}_{\mathrm{AS}}$. A mirror retroreflects the backwardpropagating Stokes and anti-Stokes modes, hence doubling the effective interaction length and allowing a single detection path consisting of a frequency-stabilized Fabry-Perot cavity (FPC) and a single-photon avalanche diode (SPAD).

and a collective spin excitation in a ${ }^{151} \mathrm{Eu}^{3+}: \mathrm{Y}_{2} \mathrm{SiO}_{5}$ crystal. This specific rare-earth-ion-doped crystal features an opticalcoherence time that can reach $2.6 \mathrm{~ms}$ [25], and a spincoherence time that can be stretched up to $6 \mathrm{~h}[26]$ under very specific experimental conditions. A drawback of rareearth ions in general, however, and even more so of $\mathrm{Eu}^{3+}$ ions in particular, is their weak oscillator strengths, which makes the off-resonant Raman excitation used in the DLCZ scheme very challenging to implement [27]. Resonant excitation, on the other hand, introduces fast inhomogeneous dephasing of the generated collective spin excitation [28], which leads to low readout efficiency of the anti-Stokes photons. A proposed solution to this dilemma is to employ inhomogeneous dephasing control on the optical transition [29], which in principle would allow efficient resonant generation of Stokes photons without reducing the memory readout efficiency. This can be done by creating an $\mathrm{AFC}$ on the optical transition, which is a technique that has been successfully employed to store quantum states of light on optical transitions [15-18].

The main features of the AFC-based DLCZ scheme [29] are shown in Fig. 1(a). A resonant write pulse excites a part of the AFC comb on the $|g\rangle \leftrightarrow|e\rangle$ transition. The basic mechanism of the AFC comb is to periodically rephase any inhomogeneous dephasing induced by the absorption of an optical pulse, which leads to an echo emission after a time $1 / \Delta$. In this scheme, however, the AFC echo of the write pulse is of no interest in itself. Instead, we consider the spontaneous emission of a Stokes photon on the $|e\rangle \leftrightarrow|s\rangle$ transition, at a time $T_{\mathrm{S}}$ within a time window of duration $\tau_{g}<1 / \Delta$. In Fig. 1(b) we present a typical photoncounting histogram showing the Stokes detections following the write pulse. As the detection gate has a duration $\tau_{g}=10 \mu \mathrm{s}$, which is much shorter than the radiative lifetime $T_{1}=1.97 \mathrm{~ms}$ [25] of the excited state $|e\rangle$, the Stokes detection probability is constant over the entire gate.

By conditioning on the detection of a single Stokes photon, the ensemble of ions is projected into a single collective spin-wave excitation of the form

$$
\left|\psi_{\mathrm{SW}}\right\rangle \propto \sum_{j=1}^{N} e^{-i 2 \pi \delta_{j} T_{\mathrm{S}}} e^{i \Delta \mathbf{k} \cdot \mathbf{r}_{j}} c_{j}\left|g_{1}, \ldots, s_{j}, \ldots, g_{N}\right\rangle
$$

Here $N$ is the total number of ions in the interaction volume [ $N=O\left(10^{12}\right)$ in our case], $\delta_{j}$ is the optical detuning of ion 
$j, \Delta \mathbf{k}=\mathbf{k}_{W}-\mathbf{k}_{S}$ is the wave vector of the spin wave, $\mathbf{r}_{j}$ is the position of ion $j$, and $c_{j}$ is the effective coupling constant of ion $j$. Importantly, the detection time $T_{\mathrm{S}}$ is encoded in the amount of optical dephasing $\exp \left(-i 2 \pi \delta_{j} T_{\mathrm{S}}\right)$ that has occurred before the emission. This is the fundamental reason why the scheme is temporally multimode; the detection of time-separated Stokes photons leads to distinguishable spin waves.

In order to extend the storage time, we manipulate the spin waves using radio-frequency pulses (see Fig. 1 and Supplemental Material for more details [30]). Finally the stored excitation is mapped out by applying a read pulse on the $|g\rangle \leftrightarrow|e\rangle$ transition, see Fig. 1(a). The resulting emission on the $|e\rangle \leftrightarrow|s\rangle$ transition, which we denote antiStokes photons, is detected using the same setup as for the Stokes photons, as shown in Fig. 1(c). In the experimental histogram shown in Fig. 1(b), the anti-Stokes detection probability is slightly larger than in the Stokes gate due to different sources of noise (see Supplemental Material [30]).

By measuring the coincidences between the Stokes and anti-Stokes photons, we can calculate the second-order cross-correlation function $g_{\mathrm{S}, \mathrm{AS}}$ of the photon pair [32]. If the average photon number is much less than 1 per mode, then $g_{\mathrm{S}, \mathrm{AS}}$ can be written as

$$
g_{\mathrm{S}, \mathrm{AS}} \simeq \frac{p_{\mathrm{S}, \mathrm{AS}}}{p_{\mathrm{acc}}}
$$

where $p_{\mathrm{S}, \mathrm{AS}}$ is the coincidence detection probability and $p_{\text {acc }}$ is the accidental coincidence probability due to uncorrelated photons. The AFC-based DLCZ scheme produces specific time correlations due to the AFC rephasing mechanism. The read pulse will lead to a collective optical excitation that will continue to undergo inhomogeneous dephasing, due to the phase factor $\exp \left[-i 2 \pi \delta_{j}\left(T_{\mathrm{S}}+t^{\prime}\right)\right]$ of each atom $j$ where $t^{\prime}$ is the time elapsed after the read pulse. The periodic AFC structure will ensure, however, that all atoms are in phase when $T_{\mathrm{S}}+t^{\prime}=1 / \Delta$, such that the emission probability of an anti-Stokes photon is strongly peaked for $t^{\prime}=1 / \Delta-T_{\mathrm{S}}$.

In Fig. 2(a) we show the Stokes-anti-Stokes coincidence histogram as a function of the delay $\tau=T_{\mathrm{S}}+T_{\mathrm{AS}}$, where $T_{\mathrm{AS}}=T_{\text {spin }}+t^{\prime}$. As expected, there is a strong temporal correlation at $\tau=T_{\text {spin }}+1 / \Delta=1020 \mu \mathrm{s}$. The triangular background of accidental coincidences stems from the convolution of the two square-shaped detection gates, see Fig. 2(a). To calculate $p_{\text {acc }}$ for a given delay $\tau$, we need to consider all combinations of Stokes and anti-Stokes detections that could lead to an accidental coincidence at $\tau=T_{\mathrm{S}}+T_{\mathrm{AS}}$. By normalizing with this triangular-shaped $p_{\text {acc }}$ function, we obtain the cross-correlation function $g_{\mathrm{S}, \mathrm{AS}}$, shown in Fig. 2(b). For the central bin this correlation function peaks at the value of $4.2 \pm 0.5$. The coherence time of the Stokes-anti-Stokes photon pair with respect to the detection gate shows that correlations in more than 10 modes are generated, see Fig. 2(b).

In order to verify the nonclassical nature of the Stokes-antiStokes correlations, one can use that for classical fields the cross-correlation function is bounded by the Cauchy-Schwarz inequality $R=g_{\mathrm{S}, \mathrm{AS}}^{2} /\left(g_{\mathrm{S}, \mathrm{S}} \cdot g_{\mathrm{AS}, \mathrm{AS}}\right) \leq 1$ [32], where $g_{X, X}$ $(X=\mathrm{S}$ or AS $)$ are the autocorrelation functions for each field.

We performed a simultaneous measurement of both the auto- and cross-correlation functions using $T_{\text {spin }}=500 \mu \mathrm{s}$ and $p_{\mathrm{S}}=0.002$, with $p_{\mathrm{S}}$ the probability to emit at least one Stokes photon. We obtained $g_{\mathrm{S}, \mathrm{S}}=1.86 \pm 0.4$, $g_{\mathrm{AS}, \mathrm{AS}}=1.96 \pm 0.36$, and $g_{\mathrm{S}, \mathrm{AS}}=3.24 \pm 0.43$. The given results are calculated using a bin size of $100 \mathrm{~ns}$. The violation of the Cauchy-Schwarz inequality $R=$ $2.88_{-1.4}^{+2.9}>1$ clearly demonstrates the nonclassical nature of the Stokes-anti-Stokes correlations.
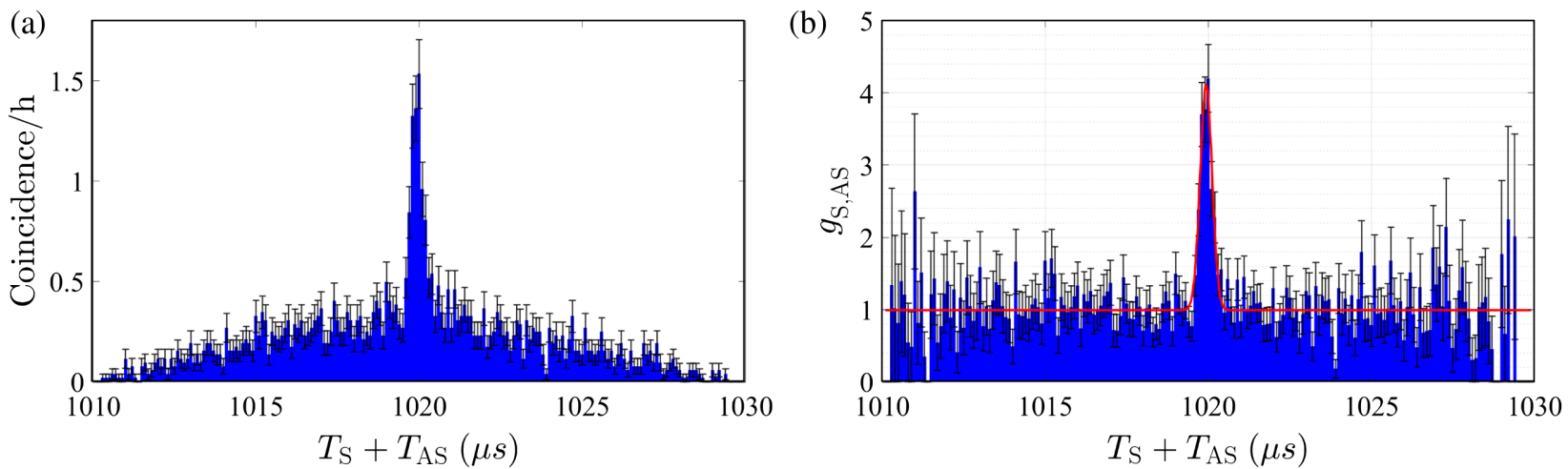

FIG. 2. (a) The Stokes-anti-Stokes coincidence detection histogram and (b) the normalized second-order cross-correlation function $g_{\mathrm{S}, \mathrm{AS}}$, as a function of $\tau=T_{\mathrm{S}}+T_{\mathrm{AS}}$. The Stokes and anti-Stokes detection gates were set to $10 \mu$ s and all detection events were binned into $100 \mathrm{~ns}$. The coincidence histogram in (a) shows the expected peak at $\tau=T_{\text {spin }}+1 / \Delta=1020 \mu$ s. The cross-correlation peak in (b) is fitted using a Gaussian function (red curve), which results in full width at half maximum of $0.41 \mu$ s coherence time of the Stokesanti-Stokes photon pair. This is in good agreement with the square excitation bandwidth of $2 \mathrm{MHz}$ of the write pulse. If we define the duration of one mode as $2 \tau_{c}$, then the AFC-based DLCZ source generates correlated Stokes-anti-Stokes pairs in $10 \mu s /\left(2 \tau_{c}\right)=12$ modes per experimental run. 
We can analyze the generation of quantum correlations in terms of a photon pair source. As a model we use the two-mode squeezed vacuum state [1,32], which is fully described by $p_{\mathrm{S}}$. We also include a finite readout efficiency of the anti-Stokes photon $\eta_{R}$, conditioned on the detection of a Stokes photon, and a source of uncorrelated noise in the anti-Stokes mode with probability $p_{n}$. Using this simple model, the $g_{\mathrm{S}, \mathrm{AS}}$ function is given by (see Supplemental Material [30])

$$
g_{\mathrm{S}, \mathrm{AS}}=1+\frac{\eta_{R}}{\left(\eta_{R}+\beta\right) p_{\mathrm{S}}+p_{n}} .
$$

The term $\beta p_{\mathrm{S}}$ accounts for spontaneous emission in the anti-Stokes gate that depends on the write pulse. Here $\beta$ is the fraction of population in the excited state at the time of the anti-Stokes emission, with respect to the initial population after the write pulse. We emphasize that $p_{n}$ and $\eta_{R}$ could be calculated independently from the experimental data and $\beta$ can be calculated using known experimental and atomic parameters of $\mathrm{Eu}^{3+}: \mathrm{Y}_{2} \mathrm{SiO}_{5}$ (see Supplemental Material [30]). As shown in Fig. 3, the theoretical model predicts very well the cross-correlation measurement as a function of the Stokes emission probability $p_{\mathrm{S}}$, without any further adjustment of the parameters.

The measured anti-Stokes efficiency of $\eta_{R}=0.45 \%$ used in the model shown in Fig. 3 refers to the efficiency in a temporal bin of $100 \mathrm{~ns}$, which is much shorter than the coherence time of the Stokes-anti-Stokes photon pair $\tau_{c}=0.41 \mu$ s (see Fig. 2). The temporal bin duration of $100 \mathrm{~ns}$ was found to represent the best compromise between the readout noise $p_{n}$ and the efficiency $\eta_{R}$, leading to the highest cross-correlation $g_{\mathrm{S}, \mathrm{AS}}$. However, if we

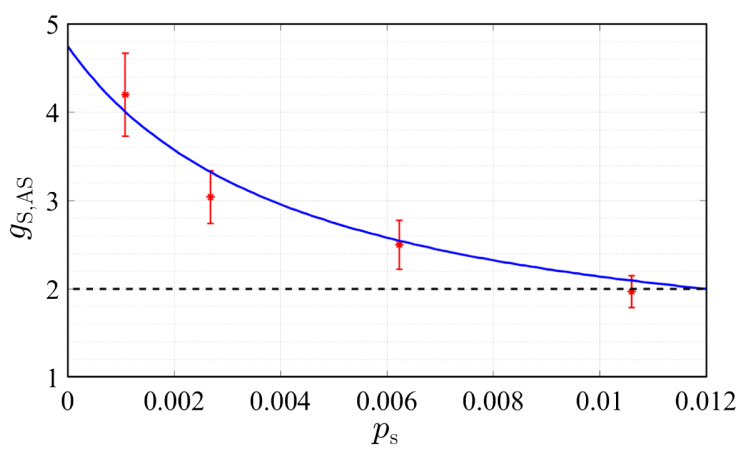

FIG. 3. Measured cross-correlation parameter $g_{\mathrm{S}, \mathrm{AS}}$ (red stars) as a function of the Stokes probability $p_{\mathrm{S}}$, for the central bin of $100 \mathrm{~ns}$ at $\tau=T_{\mathrm{S}}+T_{\mathrm{AS}}$ shown in Fig. 2(b). The theoretical model, see Eq. (3), is in close agreement with the experimental data (blue line). The model parameters were $p_{n}=0.12 \%$, $\beta=0.27$, and $\eta_{R}=0.45 \%$, all given for a bin size of $100 \mathrm{~ns}$. Note that the parameters were measured independently and not fitted (see text for details). For an ideal two-mode squeezed state $g_{\mathrm{S}, \mathrm{AS}}$ is bounded by 2 ; however, the observed violation of the Cauchy-Schwarz inequality presents a more stringent test of the nonclassical nature of the correlations. consider a temporal bin of duration $2 \tau_{c}$, then the measured anti-Stokes readout efficiency is $2.5 \pm 0.3 \%$. Our theoretical modeling based on the theory presented in [29] gives an estimated efficiency between $5 \%$ and $10 \%$, depending on assumptions in the model parameters. The discrepancy with the experiment is currently not understood, but could be due to experimental issues, such as phase-matching problems, or due to wrong assumptions in the theoretical modeling. Moreover, the measured cross-correlation was the same for the two storage times used in this work, $T_{\text {spin }}=0.5$ and $1 \mathrm{~ms}$, while based on previous measurements [5] we would have expected an increased efficiency and cross-correlation function for the shorter spin-storage time. Future experiments should investigate the readout efficiency in more detail, in particular as a function of spinstorage time. An interesting approach in this context is to use a cavity-enhanced scheme, which resulted in an efficiency increase of a factor of 10 in AFC spin-wave storage experiments [33]. Also, Zhong et al. [18] have shown that a coherence time of up to $6 \mathrm{~h}$ can be achieved in this crystal by applying a precisely aligned and strong magnetic field.

In conclusion, we have experimentally demonstrated a new multimode DLCZ scheme for generating nonclassical correlations between a single photon and a collective spin excitation in a solid-state ensemble. The key feature of the scheme is the use of an atomic frequency comb to control the inhomogeneous dephasing, which allowed us to generate spin-photon correlations in at least 12 temporal modes. Even more modes could, in principle, be generated by increasing the delay $1 / \Delta$, or by increasing the bandwidth of the AFC. Neither of these two parameters have reached their intrinsic limit in the specific case of ${ }^{151} \mathrm{Eu}^{3+}: \mathrm{Y}_{2} \mathrm{SiO}_{5}$ [4]. The readout efficiency was low in the present demonstration, which in combination with the technical photon noise source limited the observed crosscorrelation function. This is not a fundamental issue, however, as noise can be reduced by further optimizing the spectral filtering elements and the efficiency can be boosted by a cavity approach, as we recently demonstrated [33]. The ability to generate multimode quantum correlations with long storage times is a key requirement for realizing quantum repeaters for long-distance quantum communication. Therefore, we believe our results represent an important step for realizing a quantum repeater based on solid-state ensembles.

The authors thank Genko Genov, Nuala Timoney, and Anthony Martin for useful discussions, as well as Claudio Barreiro for technical support. We acknowledge funding from the Swiss program National Centres of Competence in Research (NCCR) project Quantum Science Technology (QSIT), the EU's H2020 program under the Marie Skodowska-Curie project Quantum Communications for ALL (QCALL Grant No. GA 675662), and the EU's FP7 
programme under the ERC Advanced Grant Macroscopic Entanglements in Crystals (MEC Grant No. GA 339198).

Note added in proof-A related experiment in a Praseodymium-doped crystal has been performed in parallel [34].

*Present address: Institut für Experimentalphysik, Universität Innsbruck, Technikerstraße 25, 6020 Innsbruck, Austria. †mikael.afzelius@unige.ch

[1] L.-M. Duan, M. D. Lukin, J. I. Cirac, and P. Zoller, Nature (London) 414, 413 (2001).

[2] H. J. Kimble, Nature (London) 453, 1023 (2008).

[3] N. Sangouard, C. Simon, H. de Riedmatten, and N. Gisin, Rev. Mod. Phys. 83, 33 (2011).

[4] P. Jobez, N. Timoney, C. Laplane, J. Etesse, A. Ferrier, P. Goldner, N. Gisin, and M. Afzelius, Phys. Rev. A 93, 032327 (2016).

[5] P. Jobez, C. Laplane, N. Timoney, N. Gisin, A. Ferrier, P. Goldner, and M. Afzelius, Phys. Rev. Lett. 114, 230502 (2015).

[6] T. Chanelière, D. N. Matsukevich, S. D. Jenkins, S.-Y. Lan, T. A. B. Kennedy, and A. Kuzmich, Nature (London) 438, 833 (2005).

[7] M. D. Eisaman, A. André, F. Massou, M. Fleischhauer, A. S. Zibrov, and M. D. Lukin, Nature (London) 438, 837 (2005).

[8] S.-J. Yang, X.-J. Wang, X.-H. Bao, and J.-W. Pan, Nat. Photonics 10, 381 (2016).

[9] C. W. Chou, H. de Riedmatten, D. Felinto, S. V. Polyakov, S. J. van Enk, and H. J. Kimble, Nature (London) 438, 828 (2005).

[10] C. W. Chou, J. Laurat, H. Deng, K. S. Choi, H. de Riedmatten, D. Felinto, and H. J. Kimble, Science 316, 1316 (2007).

[11] S.-Y. Lan, A. G. Radnaev, O. A. Collins, D. N. Matsukevich, T. A. Kennedy, and A. Kuzmich, Opt. Express 17, 13639 (2009).

[12] H. Bernien, B. Hensen, W. Pfaff, G. Koolstra, M. S. Blok, L. Robledo, T. H. Taminiau, M. Markham, D. J. Twitchen, L. Childress, and R. Hanson, Nature (London) 497, 86 (2013).

[13] A. Delteil, Z. Sun, W.-b. Gao, E. Togan, S. Faelt, and A. Imamoğlu, Nat. Phys. 12, 218 (2016).

[14] R. Riedinger, S. Hong, R. A. Norte, J. A. Slater, J. Shang, A. G. Krause, V. Anant, M. Aspelmeyer, and S. Gröblacher, Nature (London) 530, 313 (2016).

[15] C. Clausen, I. Usmani, F. Bussieres, N. Sangouard, M. Afzelius, H. de Riedmatten, and N. Gisin, Nature (London) 469, 508 (2011).
[16] D. Rielander, K. Kutluer, P. M. Ledingham, M. Gundogan, J. Fekete, M. Mazzera, and H. de Riedmatten, Phys. Rev. Lett. 112, 040504 (2014).

[17] E. Saglamyurek, J. Jin, V. B. Verma, M. D. Shaw, F. Marsili, S. W. Nam, D. Oblak, and W. Tittel, Nat. Photonics 9, 83 (2015).

[18] Z.-Q. Zhou, Y.-L. Hua, X. Liu, G. Chen, J.-S. Xu, Y.-J. Han, C.-F. Li, and G.-C. Guo, Phys. Rev. Lett. 115, 070502 (2015).

[19] K. R. Ferguson, S. E. Beavan, J. J. Longdell, and M. J. Sellars, Phys. Rev. Lett. 117, 020501 (2016).

[20] W. Tittel, M. Afzelius, T. Chanelière, R. Cone, S. Kröll, S. Moiseev, and M. Sellars, Laser Photonics Rev. 4, 244 (2010).

[21] Y.-W. Cho, G. T. Campbell, J. L. Everett, J. Bernu, D. B. Higginbottom, M. T. Cao, J. Geng, N. P. Robins, P. K. Lam, and B. C. Buchler, Optica 3, 100 (2016).

[22] M. Afzelius, C. Simon, H. de Riedmatten, and N. Gisin, Phys. Rev. A 79, 052329 (2009).

[23] B. Albrecht, P. Farrera, G. Heinze, M. Cristiani, and H. de Riedmatten, Phys. Rev. Lett. 115, 160501 (2015).

[24] A. E. Ulanov, I. A. Fedorov, A. A. Pushkina, Y. V. Kurochkin, T. C. Ralph, and A. I. Lvovsky, Nat. Photonics 9, 764 (2015).

[25] F. Könz, Y. Sun, C. W. Thiel, R. L. Cone, R. W. Equall, R. L. Hutcheson, and R. M. Macfarlane, Phys. Rev. B 68, 085109 (2003).

[26] M. Zhong, M. P. Hedges, R. L. Ahlefeldt, J. G. Bartholomew, S. E. Beavan, S. M. Wittig, J. J. Longdell, and M. J. Sellars, Nature (London) 517, 177 (2015).

[27] E. A. Goldschmidt, S. E. Beavan, S. V. Polyakov, A. L. Migdall, and M.J. Sellars, Opt. Express 21, 10087 (2013).

[28] C. Ottaviani, C. Simon, H. de Riedmatten, M. Afzelius, B. Lauritzen, N. Sangouard, and N. Gisin, Phys. Rev. A 79, 063828 (2009).

[29] P. Sekatski, N. Sangouard, N. Gisin, H. de Riedmatten, and M. Afzelius, Phys. Rev. A 83, 053840 (2011).

[30] See Supplemental Material at http://link.aps.org/ supplemental/10.1103/PhysRevLett.118.210501, which includes Ref. [31], for a description of experimental details and a brief discussion on the theoretical model for the crosscorrelation function.

[31] M. Tian, T. Chang, K. D. Merkel, and W. R. Babbitt, Appl. Opt. 50, 6548 (2011).

[32] C. W. Chou, S. V. Polyakov, A. Kuzmich, and H. J. Kimble, Phys. Rev. Lett. 92, 213601 (2004).

[33] P. Jobez, I. Usmani, N. Timoney, C. Laplane, N. Gisin, and M. Afzelius, New J. Phys. 16, 083005 (2014).

[34] K. Kutluer, M. Mazzera, and H. de Riedmatten, following Letter, Phys. Rev. Lett. 118, 210502 (2017). 